\title{
Research on Parameters Optimization of NC Gear Hobbing Process Based on Box-Behnken Design
}

\author{
Weilong Yi
}

\begin{abstract}
Driven by the development of numerical control technology, the gear manufacturing industry develops towards the direction of green, low carbon and stable quality. However, process parameters of numerical control gear hobbing machine is too conservative at present, which does not fully exploit its outstanding performance. Therefore, it is of great significance to study the parameters optimization of NC gear hobbing process. This paper first analyzes the factors affecting carbon emission and precision in hobbing process. Then, Box-Behnken Design is carried out for this process and the BP neural network model is established based on the experimental data. Finally, the Particle Swarm Optimization algorithm is applied to solve the model and three groups of optimal process parameters combination are obtained, which consist of minimum carbon emission, optimal precision and comprehensive optimization.
\end{abstract}

Keywords-NC gear hobbing, parameters optimization, carbon emission, precision.

\section{INTRODUCTION}

As an important component in a complex mechanical transmission system, gears are widely used in transportation, wind power generation and other industries. The NC gear hobbing process is complex and generally undergoes three stages of processing before, during and after heat treatment, including a series of processes such as turning, gear hobbing, and gear shaving. Among them, the gear hobbing stage has high energy consumption and great influence on the precision of gear forming.

Numerous studies have shown that the selection of parameters in the manufacturing process will have different effects on energy consumption and precision. Therefore, it is important to reduce carbon emissions and improve precision by optimizing parameters.

Newman et al. [1] designed related experiments for the milling process and the study showed that reasonable changes in cutting parameters can reduce the energy consumption by up to $40 \%$. For the turning process, Velchev et al. [2] first conducted experiments to collect turning data, then established an energy model and determined the key coefficients. Kant et al. [3] established a correlation model between lathe energy consumption and product roughness, and used the response surface method to quantify the interactive effects of different turning parameters. Campateili et al. [4] designed a response

Manuscript received May. 10, 2021. This work was supported in part by the National Key Technology R\&D Program of China (2018YFB1701205), and the National Natural Science Foundation of China (5200051148).

Weilong Yi is with the State Key Laboratory of Mechanical Transmission, Chonqing University, Chongqing, China surface test for the milling process to collect milling process parameters and milling machine energy consumption data, then Through regression analysis, the relevant factors that significantly affect the energy consumption of milling machine, namely milling width and milling feed, are selected. For the turning process, Bilga et al. [5] found that the depth of cut is the most significant factor affecting energy efficiency through Taguchi orthogonal test and signal-to-noise ratio analysis. Kumar et al. [6] applied the analytic hierarchy process to determine that the factor that significantly affects energy consumption and precision is the cutting speed. Sun et al. [7] optimized the hobbing process parameters with the smallest tooth profile error as the optimization goal, which effectively improved the precision of gear hobbing process.

At present, the adjustment of process parameters mainly includes querying the processing manual, developing corresponding software module, and relying on experienced operators. No matter which method is adopted, the efficiency of process parameter optimization is relatively low. Data science has developed well and research methods have been constantly changing from physical modeling to data-driven modeling in recent years. The use of data-driven methods to solve complex engineering problems has become a research hotspot.

Zhang et al. [8] applied data-driven methods to wastewater pumping systems, using neural networks and artificial immune algorithms to reduce system energy consumption. Xiao et al. [9] combined data mining and fuzzy logic theory to optimize the energy efficiency and time of the turning system. Zhong et al. [10] used the BP neural network to establish a prediction model of time and energy consumption for the high-speed dry cutting gear hobbing process, and used the flower pollination algorithm to optimize the process parameters. Lu et al. [11] successfully predicted the energy consumption of gear processing by collecting data of design, manufacturing and energy consumption, which laid a certain foundation for energy saving.

Above researches on process parameters optimization have made certain contributions in reducing carbon emissions and energy consumption. However, most of them only focus on simple machining processes such as turning and milling. There are many difficulties in determining reasonable parameters on account of the numbers and ranges of parameters.

For this reason, this paper uses data-driven method to optimize the parameters of $\mathrm{NC}$ gear hobbing process from carbon emissions and precision aspects. First, the factors influencing carbon emission and precision of gear hobbing are analyzed, on this basis, the Box-Behnken Design (BBD) 
method is used to design a test of input variables and output indicators. Then, the BP neural network model is established to predict the carbon emission and precision of gear hobbing based on the data. Finally, a process parameter optimization model with the lowest carbon emission and optimal precision is built,and the particle swarm algorithm (Particle Swarm Optimization algorithm (PSO)) is used to solve the model. As a result, three sets of optimal process parameters including lowest carbon emission, optimal precision and comprehensive optimization are obtained.

\section{ANALYSIS OF INFLUENCING FACTORS OF NC GEAR HOBBING PROCESS}

\section{A. Analysis of influencing factors of carbon emission}

Materials, auxiliary materials, energy consumption and waste disposal will affect the carbon emissions of the machining system [12]. This section analyzes the influencing factors of carbon emission of gear hobbing process from the above perspective.

1) Impact of machine tool equipment

In the process of gear hobbing, various operating parameters of NC gear hobbing machine tools will change in different stages. Specifically, the gear hobbing machine tool has a complex structure, including a processing system and auxiliary system. In different stages of gear hobbing, changes in motor parameters will cause changes in hobbing speed and hobbing feed. During this process, the carbon emissions produced by the processing system change continuously over time, which can be calculated by integrating the power curve. The power of the auxiliary system varies little with the processing stage, so it can be regarded as unchanged in the time series, and its carbon emissions can be calculated by multiplying the power by the time.

2) Impact of materials and auxiliary materials

In the hobbing process, materials and auxiliary materials (such as coolant, lubricating oil, etc.)would be consumed, and they produced carbon emissions during their own preparation process, so the calculation of rolling Tooth carbon emissions should also be taken into consideration. The exact carbon emission of materials and auxiliary materials in the preparation process can not be known in advance, so it can be evaluated by the following methods: firstly, the materials and auxiliary materials consumed can be calculated through the comparison before and after the process; secondly, the carbon emission factor table of common materials can be consulted to determine the carbon emission factor; finally, the specific materials can be converted into standard coal for conversion.

3) Impact of waste disposal

After the waste generated by the gear hobbing processing system is collected, removed from impurities, briquetting, etc., it needs to be refurbished, and carbon emissions will also be generated in this process. Generally speaking, the commonly used materials for tooth blanks mainly include forged steel, cast steel and cast iron, which can be recycled and reused after degreasing, drying, and briquetting. Due to different reheating standards, the carbon emissions of different materials and wastes are also different. The method of assessing waste carbon emissions is the same as previously explained. The central idea is to calculate waste carbon emission factors and the amount of standard coal consumed to process these wastes.

\section{B. Analysis of factors affecting precision}

Gear hobbing is one of the core processes in the gear shaping process. In the process of gear hobbing, the process system composed of machine tool-workpiece-tool will affect gear precision.

1) Impact of machine tool equipment

As the actuator of gear hobbing, the machine tool's own error will inevitably affect the precision of the gear. Generally speaking, machine tool errors are mainly divided into guide rails, spindles, and transmission chain errors according to different parts. The accumulation and transmission of various errors will change the relative positional relationship between the hob and the gear blank. As a result, they will reduce gear precision.

\section{2) Impact of the hob}

The gear hobbing is the process in which the hob and the gear blank mesh with each other. Therefore, when the state of the hob changes, the quality of the gear will also be affected. Specifically, the state of the hob mainly includes changes in hob wear, damage, runout, and movement. The wear of the hob will increase the chip resistance, which will further reduce the hob cutting performance and reduce the gear precision. The destruction will cause the tool to be replaced, which indirectly affects its installation precision; the runout and movement will make the size of the machined gear uneven and increase the tooth profile error.

3) Impact of the tooth blank

The gear blank will also have a certain impact on the precision of hobbing. Due to the uneven geometry and material hardness of the gear blank, the cutting force will be different during the cutting process. As a result, the relative positional relationship got affected and the precision got reduced.

4) Impact of process parameters

In the process of gear hobbing, cutting process parameters will have a significant impact on the efficiency and quality of gear hobbing. Therefore, it is very important to select reasonable cutting process parameters. In the gear hobbing process, the cutting process parameters mainly include the hobbing depth, the hobbing speed and the hobbing feed. Cutting process parameters indirectly affect the rigidity and stability of the process system by influencing the hobbing force, thereby changing the cutting state of the hob and gear blank and ultimately affecting the gear precision.

The increase of the hobbing depth will make the hobbing force getting increase in a subtle way. This is because the hobbing depth is closely related to the gear shape (tooth profile correction coefficient). When the value is small, the effect on the hobbing force is relatively small. The hobbing speed is related to the friction between the hob and the gear, so it will also affect the hobbing force. When processing plastic materials, the hobbing force will increase with the increase of 
the hobbing speed in the low-speed range, while the hobbing force will decrease with the increase of the hobbing speed since the temperature of the cutting zone got increased, resulting in the decrease in material strength and friction coefficient.

The increase of the hobbing feed will increase the hobbing force. Compared with the hobbing depth, the change of the hobbing feed under the same conditions has less influence on the hobbing force.

\section{A comprehensive analysis of influencing factors}

There are many factors that affect gear precision, mainly composed of machine tools, tools, gears, and machining cutting parameters. Among them, machining and cutting parameters involve the most variables. The factors that affect gear hobbing carbon emissions mainly include material consumption, auxiliary material consumption, electrical energy consumption and waste disposal, among which electric energy consumption involves the most variables.

In actual production activities, according to the requirements of specific processing tasks, the strength and load-bearing capacity of the product itself, and other related requirements limit the parameters of the tooth blank workpiece and the performance parameters of the machine tool. In addition, in order to ensure the processing plan, the company has also made corresponding constraints on the number of hob heads and the diameter of the hob when purchasing tools. Therefore, the machine performance parameters, tool parameters and gear workpiece parameters are set unchanged. Cutting parameters are the main factors affecting gear precision.

When gear hobbing, carbon emission sources are mainly composed of materials, auxiliary materials, electricity and waste treatment. For this study, the quality of each gear blank is approximately the same, and the quality of the processed product is almost the same. From the law of conservation of quality, it can be known that the material consumption and waste disposal carbon emissions remain unchanged. In the case of the same process route, we have sufficient reasons to assume that the consumption of auxiliary materials will also remain unchanged. Therefore, this study only regards electricity consumption as the main influencing factor of gear hobbing carbon emissions.

The key factors affecting hobbing precision and gear hobbing carbon emissions are not independent of each other, and some of these factors will interact. In actual gear hobbing, the following factors have a significant impact on gear hobbing precision and carbon emissions.

1) Depth of cut

For gears with a small modulus and a small number of teeth, the depth of cut is equal to the tooth width in actual hobbing. No matter from the point of view of precision or carbon emission, it will not change during the same batch of the same gear hobbing. . In this case, it can be considered that the depth of cut will not affect the gear hobbing precision and gear hobbing carbon emissions, so this study ignores its actual impact.
2) Machine running time and running power

There is a close correlation between machine tool running time and running power. The invalid time and power fluctuate in a small range due to the similarity of the process, and can be approximately regarded as constant. Machine cutting time and cutting power can actually be determined according to different combinations of cutting depth, hobbing feed, and hobbing speed.

Based on the above theoretical analysis, combined with the actual machining environment, this reaserch divides the gear hobbing into two stages: rough rolling and fine rolling. It is determined that the independent variables affecting gear hobbing process are rough rolling feed rate, rough rolling speed, fine rolling feed rate and fine rolling speed. The dependent variables are gear hobbing precision and gear hobbing carbon emission.

\section{BOX-BEHNKEN DESIGN THEORY}

Experimental design is a scientific method for multiple factors and corresponding response variables. A good experimental design can obtain the relationship between factors and response variables in a low-cost and high-efficiency way. With excellent nonlinear evaluation capability of input factors and corresponding response variables and the reasonable range of the parameter, Box-Behnken Design is widely used.

The design points of Box-Behnken Design include cubic points and central points. Cubic points correspond to the maximum and minimum of two level factors, which represent the boundary of the change range of input parameters. Central points are composed of the average value of the input parameters. To show the design point clearly, Fig. 1 is used as an example to illustrate the example of two level factors.

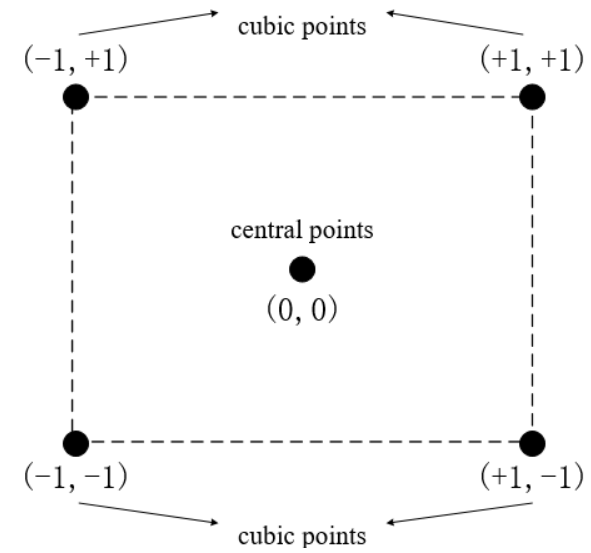

Fig. 1 The design points of Box-Behnken Design

The advantages of Box-Behnken Design include: a). the ability of evaluating non-linear effects. b). higher test safety. c). strong search ability of optimal factor level. d). fewer tests.

\section{BP NeURAL NeTwork Modeling}

The working principle of Back-Propagation-Network is to use a set of given input values and output values as training 
data and iterating from forward propagation and backward error calculation. While calculating the error between the predicted value and the actual value, the weight and bias of the neural network are continuously updated in the direction of gradient descent until the error reaches the requirement or the maximum number of iterations.

BP neural network generally consists of multiple layers, including input layer, hidden layer and output layer. Different layers have multiple nodes and the specific number is determined by research content. Figure. 2 shows the structure of 3-layer classic BP neural network, where $x_{1}, x_{2}, \ldots, x_{n}$ are input parameters, $w_{1 j}, w_{i j}, \ldots w_{n j}$ are neural network weights, $I_{1}, I_{2}, \ldots, I_{t}$ are parameters of hidden layers, $O_{1}, O_{2}, \ldots, O_{k}$ are output parameters.

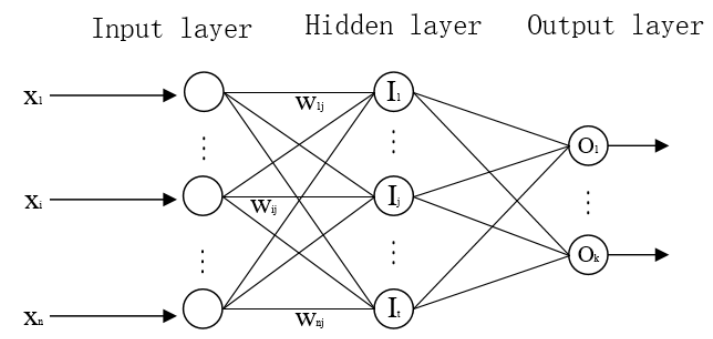

Fig.2 The structure of 3-layer classic BP neural network

BP neural network establishment steps are as follows:

Initialize the weights and biases of the hidden layers of the network. Since there are no special requirements, they are randomly selected from -1 to +1 .

According to the network structure from the input layer to the hidden layer, the calculation formula is as follows:

$$
I_{j}=\sum_{i=1}^{n} W_{i j} O_{i}+\theta_{j}
$$

In the formula, $O_{i}$ is the input value, and $\theta_{j}$ is the transfer direction of the jth neuron in the hidden layer. Considering the activation function, the actual output of the hidden layer is calculated as follows:

$$
O_{j}=\mathrm{f}\left(I_{j}\right)=\frac{1}{1+e^{-I j}}
$$

In the formula, $\mathrm{f}$ is the excitation function. In the same way, the actual output value of the output layer can be obtained.

Calculate the error of the output layer (between the actual value and the predicted value), the calculation formula is as follows:

$$
E_{k}=O_{k}\left(1-O_{k}\right)\left(T_{k}-O_{k}\right)
$$

In the formula, $T_{k}$ is the actual value, and $O_{k}$ is the predicted value.

The error calculation formula of the hidden layer is as follows:

$$
E_{j}=O_{j}\left(1-O_{j}\right) \sum_{k} E_{k} W_{j k}
$$

In the formula, $O_{j}$ is the output value of the hidden layer, $E_{k}$ is the calculation error of the output layer, and $W_{j k}$ is the weight of the neuron between the hidden layer and the output layer.

The weight and bias will be updated as the iteration progresses. The calculation formula is as follows:

$$
\Delta w_{i j}=\lambda E_{j} O_{i}
$$

$$
\begin{aligned}
& W_{i j}=W_{i j}+\Delta w_{i j} \\
& \Delta \theta_{j}=\lambda E_{j} \\
& \theta_{j}=\theta_{j}+\Delta \theta_{j}
\end{aligned}
$$

In the formula, $\lambda$ is the learning rate, $0<\lambda \leq 1$.

Repeat the above steps until the expected error or the maximum number of iterations is reached, and the algorithm ends.

\section{Multi-Objective Optimization Model And Solution FOR NC GEAR HOBBING}

\section{A. Multi-objective optimization model of NC gear hobbing}

In the process of product manufacturing, process designers usually take time, cost and other factors as optimization goals. As people's awareness of environmental protection has generally increased, relevant requirements for green and low-carbon must also be vigorously promoted. As a typical representative of the production process of complex parts, gear hobbing has been facing the problems of high energy consumption and high carbon emissions. Based on the above considerations, while optimizing the gear hobbing precision, this section also uses carbon emissions as an optimization variable and formulates related constraints.

For ease of expression, the input and output variables are now represented by the following symbols..

$\begin{array}{ll}\text { symbols } & \text { meaning } \\ F r r & \text { Feed of rough rolling } \\ R r S & \text { Rough rolling speed } \\ F f r & \text { Feed of fine rolling } \\ F r S & \text { Fine rolling speed } \\ C E & \text { Carbon Emissisions of hobbing } \\ F_{r} & \text { Radial runout tolerance } \\ F_{p} & \text { Cumulative total deviation of tooth pitch } \\ F_{\alpha} & \text { Total deviation of tooth profile } \\ f_{p t} & \text { Single pitch deviation } \\ F_{\beta} & \text { Total deviation of helix }\end{array}$

The input optimization variables involved in the hobbing process include $\mathrm{Frr}, \mathrm{Rrs}, \mathrm{Ffr}$ and Frs. The output optimization variables include $C E, F_{r}, F_{p}, F_{\alpha}, f_{p t}$ and $F_{\beta}$.

In order to facilitate the subsequent target optimization, the data measuring the precision of gear hobbing are now uniformly mapped to the Pre value:

$$
\begin{gathered}
\text { Pre }=\left(\frac{F_{r}-F_{r \min }}{F_{r \max }-F_{r \min }}+\frac{F_{p}-F_{p \min }}{F_{p \max }-F_{p \min }}+\frac{F_{\alpha}-F_{\alpha \min }}{F_{\alpha \max }-F_{\alpha \min }}\right. \\
\left.+\frac{f_{p t}-f_{p t \min }}{f_{\text {ptmax }}-f_{p \text { tmin }}}+\frac{F_{\beta}-F_{\beta \min }}{F_{\beta \max }-F_{\beta \min }}\right) / 5
\end{gathered}
$$

The smaller the Pre value, the higher the gear hobbing precision. Therefore, the optimization objective is simplified to $\mathbf{C E}$ and Pre. 
The parameters of gear hobbing processing will be restricted by the conditions of the gear hobbing machine tool and production process requirements. In order to make the optimization result more in line with the actual processing requirements, the hob speed, axial feed, gear surface quality, machine power, etc. should meet the following constraints:

Hob speed $\mathrm{n}: n_{\min } \ll \mathrm{n} \ll n_{\max }$, where $n_{\min }$ and $n_{\max }$ respectively represent the minimum and maximum speed allowed.

Axial feed $f_{z}: f_{\min } \ll f_{z} \ll f_{\max }, f_{\min }, f_{\max }$ respectively represent the minimum feed and maximum feed of the hobbing machine tool along the $\mathrm{Z}$ axis.

Gear surface roughness $R_{a}: R_{a}=0.312 f_{z}^{2} / r_{a}<\left[R_{a}\right], r_{a}$ is the radius of the hob tip arc, $\left[R_{a}\right]$ is the maximum allowable roughness value on the gear surface.

Machine tool spindle motor power $P_{c}: P_{c}<\eta P_{\max }, \eta$ is the effective coefficient of power, and $P_{\max }$ is the rated power of the machine tool spindle motor.

Based on the above optimization variables and constraints, the multi-objective optimization model of gear hobbing process parameters can be expressed as follows:

$$
\begin{aligned}
& \operatorname{minF}(\mathrm{Frr}, \mathrm{Rrs}, \mathrm{Ffr}, \mathrm{Frs})=(\operatorname{minCE}, \operatorname{minPre})(10) \\
& \left\{\begin{array}{c}
n_{\min } \ll \mathrm{n} \ll n_{\max } \\
f_{\min } \ll f_{z} \ll f_{\max } \\
R_{a}=\frac{0.312 f_{z}^{2}}{r_{a}}<\left[R_{a}\right] \\
P_{c}<\eta P_{\max }
\end{array}\right.
\end{aligned}
$$

\section{B. PSO algorithm solution}

Particle Swarm Optimization (PSO) is a representative of a swarm intelligence algorithm derived from natural biological populations. This algorithm is inspired by the behavior of birds looking for food, and first proposed by Kennedy and Eberhart. Set the bird's foraging situation and give the following restrictions: a. There is one and only one food in a specific area. b. The specific location of the food cannot be known in advance. c. Each bird has the ability to perceive the distance from the current location to the food. In this case, the best way to forage is to search the surrounding area of the nearest species and determine the location of the food based on the flight experience of each bird.

The PSO algorithm treats each optimization problem as a "particle". The particle continuously iterates its position in the solution space by repeatedly comparing the excellence of the individual extreme value Pbest and the group extreme value Gbest. It is worth mentioning that the former refers to the best position found by a single particle by itself, and the latter refers to the best position of the entire particle population. As long as the position of the particle changes, the corresponding fitness value must be calculated, and the current position, individual extreme value Pbest, and group extreme value Gbest should be updated in real time through comparison.

We can set this condition, $\mathrm{n}$ particles form a population in a D-dimensional solvable search space $\mathrm{X}=\left(X_{1}, X_{2}, X_{3}, \ldots, X_{n}\right)$, a D-dimensional vector $X_{i}=\left[x_{i 1}, x_{i 2}, x_{i 3}, \ldots, x_{i D}\right]^{T}$ to represent the $\mathrm{i}$-th particle in the population. At this time, according to the required objective function, a formula can be used to calculate the fitness value corresponding to each particle position $X_{i}$. Record the velocity of the i-th particle as $V_{i}=\left[V_{i 1}, V_{i 2}, V_{i 3}, \ldots, V_{i D}\right]^{T}$, and its individual extreme value is $P_{i}=\left[P_{i 1}, P_{i 2}, P_{i 3}, \ldots, P_{i D}\right]^{T}$, The extreme value of this population is $P_{g}=\left[P_{g 1}, P_{g 2}, P_{g 3}, \ldots, P_{g D}\right]^{T}$.

In the process of reciprocating changes from time to time, the individual extremum and global extremum will be updated in time, which will also drive the particle's own velocity and position to update accordingly. The specific formula is as follows:

The d-dimensional velocity update formula of particle $\mathrm{i}$ :

$V_{i d}^{k+1}=\omega V_{i d}^{k}+c_{1} r_{1}\left(P_{i d}^{k}-X_{i d}^{k}\right)+c_{2} r_{2}\left(P_{g d}^{k}-X_{i d}^{k}\right)(12)$

The d-dimensional position update formula of particle $\mathrm{i}$ :

$$
X_{i d}^{k+1}=X_{i d}^{k}+V_{i d}^{k+1}
$$

In the formula, $V_{i d}^{k+1}$ is the d-dimensional component of the flight velocity vector of the $(\mathrm{k}+1)$ th iteration particle I, $X_{i d}^{k+1}$ is the d-dimensional component of the position vector of the $(\mathrm{k}+1)$ th iteration particle $\mathrm{I}, \omega$ is a weighting factor (inertial weight), used to control the ability to search the solution space, $c_{1}$ and $c_{2}$ are non-negative constants, called learning factors (acceleration factors), the maximum span of adjustable algorithm learning, $r_{1}$ and $r_{2}$ are distributed in [ $0,1]$ Random number in the interval, used to increase the non-directionality and rationality of the search.

In addition, in order to prevent the algorithm from falling into an endless loop, the speed $V$ is limited to $\left[-V_{\max }, V_{\max }\right]$, and the position $X$ is limited to $\left[-X_{\max }, X_{\max }\right]$.

\section{CASE Study}

According to the related process requirements of $\mathrm{NC}$ gear hobbing of a certain factory, the gear material is 45 steel, the gear blank specification is $\varnothing 170 \times 62$, the modulus $M$ is $4.5 \mathrm{~mm}$, the number of teeth $\mathrm{Z}$ is 34 , the pressure angle $\alpha$ is $20^{\circ}$ and the gear precision needs to be 7 level. With reference to previous processing experience, set the level and range of input variables as follows:

Frr: 3 levels, respectively $2.0 \mathrm{~mm} / \mathrm{r}, 2.2 \mathrm{~mm} / \mathrm{r}, 2.4 \mathrm{~mm} / \mathrm{r}$

Rrs: 3 levels, namely 86m/min, 95 m/min, 104 m/min

Ffr: 3 levels, respectively $2.8 \mathrm{~mm} / \mathrm{r}, 3.1 \mathrm{~mm} / \mathrm{r}, 3.4 \mathrm{~mm} / \mathrm{r}$

Frs: 3 levels, namely $120 \mathrm{~m} / \mathrm{min}, 135 \mathrm{~m} / \mathrm{min}, 150 \mathrm{~m} / \mathrm{min}$

According to the Box-Behnken experiment design and data collection, the following table was obtained: 
Table I Data of hobbing process parameter

\begin{tabular}{|c|c|c|c|c|c|c|c|c|c|c|}
\hline Run & $\underset{\substack{\mathrm{Fr} \\
\mathrm{m} / \mathrm{r}}}{ }$ & $\underset{\mathrm{m} / \mathrm{min}}{\mathrm{Rrs}}$ & 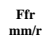 & $\underset{\substack{\text { Frs } \\
\mathrm{m} / \min }}{\operatorname{ros}}$ & $\underset{\mathrm{Kg} / \mathrm{CO} 2}{\mathrm{CE}}$ & $\underset{\substack{F_{r} \\
m}}{F_{n}}$ & $\begin{array}{c}F_{p} \\
\mathrm{~mm}\end{array}$ & $\begin{array}{c}F_{\alpha} \\
\mathrm{mm}\end{array}$ & $\begin{array}{c}f_{p t} \\
\mathrm{~mm}\end{array}$ & $\begin{array}{c}F_{\beta} \\
\text { mm }\end{array}$ \\
\hline 1 & 2 & 95 & 3.4 & 135 & 0.3796 & 38.30 & 48.70 & 23.44 & 16.46 & 20.74 \\
\hline 2 & 2.4 & 95 & 3.1 & 120 & 0.4208 & 42.05 & 53.43 & 25.02 & 17.72 & 22.63 \\
\hline 3 & 2 & 86 & 3.1 & 135 & 0.2734 & 36.82 & 47.59 & 22.67 & 16.18 & 19.82 \\
\hline & & & & & & & & $\ldots$ & & $\cdots$ \\
\hline 27 & 2.2 & 95 & 3.1 & 135 & 0.3316 & 37.27 & 47.50 & 22.97 & 16.17 & 20.17 \\
\hline 28 & 2.2 & 86 & 2.8 & 135 & 0.2651 & 35.78 & 46.52 & 22.28 & 15.90 & 19.24 \\
\hline 29 & 2.2 & 95 & 3.4 & 120 & 0.4880 & 42.97 & 54.46 & 25.62 & 17.93 & 23.15 \\
\hline
\end{tabular}

First, apply BP neural network to predict the carbon emission and precision of hobbing, the steps are as follows:

1) Determine the number of input and output nodes

In this paper, a three-layer BP neural network is used. Since Frr, Rrs, Ffr and Frs are used as input variables while CE, $F_{r}$, $F_{p}, F_{\alpha}, f_{p t}$ and $F_{\beta}$ as output variables, the input nodes of the neural network are 4 and the output nodes are 6 .

2) the results of model prediction

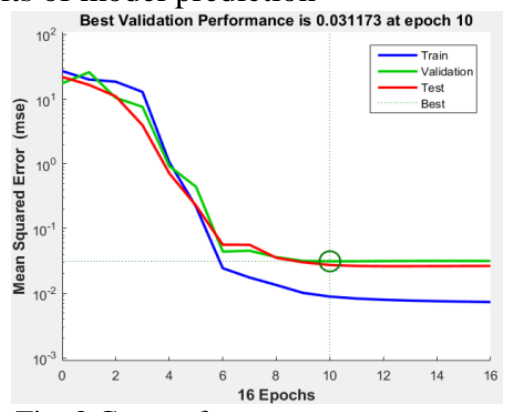

Fig. 3 Curve of root mean square error

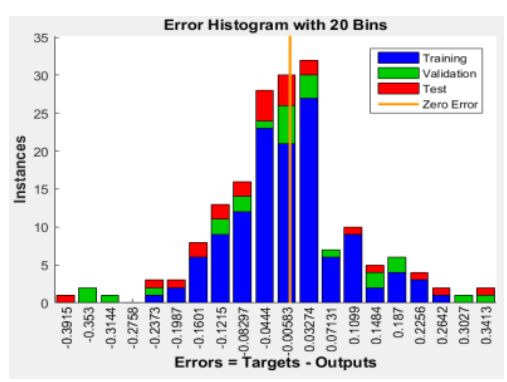

Fig. 4 Curve of error distribution

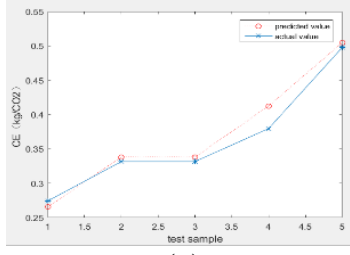

(a)

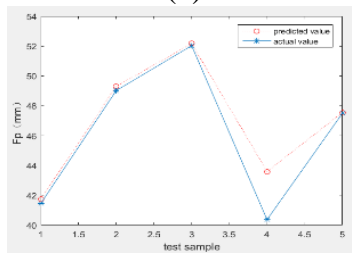

(c)

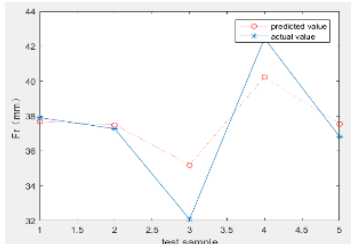

(b)

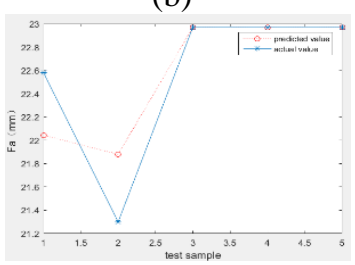

(d)

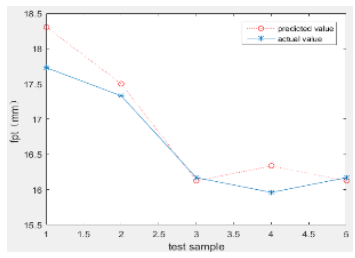

(e)

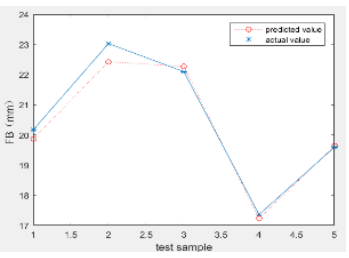

(f)
Fig. 5 Prediction error curve of BP neural network

Table II Predict error of BP neural network

\begin{tabular}{cccccccc}
\hline \hline \multirow{2}{*}{ method } & Predicted values & $\mathbf{C E}$ & $\boldsymbol{F}_{\boldsymbol{r}}$ & $\boldsymbol{F}_{\boldsymbol{p}}$ & $\boldsymbol{F}_{\boldsymbol{\alpha}}$ & $\boldsymbol{f}_{\boldsymbol{p t}}$ & $\boldsymbol{F}_{\boldsymbol{\beta}}$ \\
\hline \multirow{2}{*}{ BP } & Error value & 0.03 & 1.08 & 2.16 & 1.16 & 0.22 & 0.63 \\
& Relative error & $7.81 \%$ & $3.48 \%$ & $4.53 \%$ & $4.96 \%$ & $1.36 \%$ & $3.26 \%$ \\
\hline \hline
\end{tabular}

It can be seen from table 2 that BP neural network has a good effect on carbon emission prediction and precision prediction of NC gear hobbing.

Then, realize model optimization using PSO algorithm.

The BP algorithm is a process of optimizing weights and biases. The biggest problem it has is that it is easy to fall into a local optimum, while the PSO algorithm can make up for this shortcoming. The PSO algorithm is a process of global optimization, which can randomly generate multiple particles within a specified space, and then use these particles to search the surrounding space. The optimization of the PSO algorithm to the BP neural network is mainly reflected in the optimization of the fitness function corresponding to the objective function. When the fitness function reaches the minimum value, the error of the objective function will also be minimized, thereby updating the weight and bias of the network. The optimization process is shown in Figure. 6:

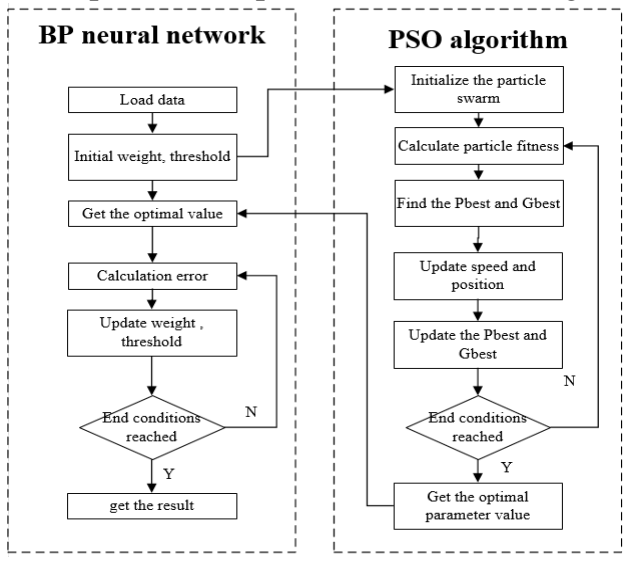

Fig. 6 flow chart of PSO-BP

In the process of gear hobbing, gear hobbing carbon emissions and precision are factors that need to be optimized. However, in the multi-objective optimization problem, most of the different objectives will conflict with each other, so it is difficult to obtain the optimal value of multiple objectives at the same time. The solution is to transform it into a single-objective optimization problem through linear weighting, which is a method of making decisions first and then searching for the best. Considering the low-carbon demand and precision requirements of actual processing, three sets of influence weights of minimum carbon emission, 
optimal precision and comprehensive optimization are respectively set to optimize the combination of process parameters.

The change of the optimal individual fitness value is shown in Figure. 7.

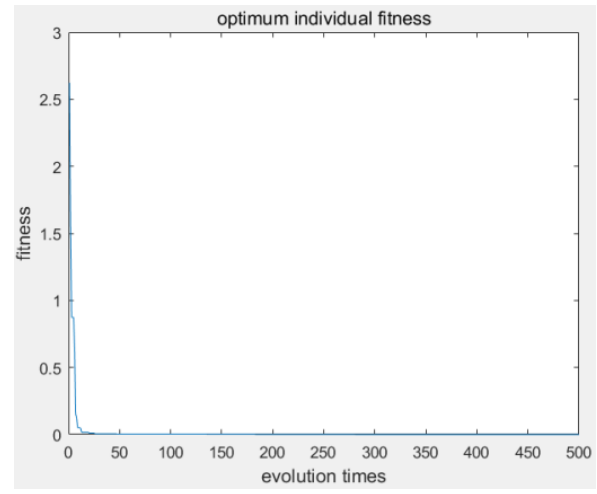

Fig. 7 Fitness value change of optimal individual

Table III Comparison table of optimization results

\begin{tabular}{cccccccc}
\hline \hline $\begin{array}{c}\text { Sche } \\
\mathbf{m e}\end{array}$ & $\begin{array}{c}\text { Optimization } \\
\text { objective }\end{array}$ & $\begin{array}{c}\mathbf{F r r} \\
\mathbf{m m} / \mathbf{r}\end{array}$ & $\begin{array}{c}\mathbf{R r s} \\
\mathbf{m} / \mathbf{m i n}\end{array}$ & $\begin{array}{c}\mathbf{F f r} \\
\mathbf{m m} / \mathbf{r}\end{array}$ & $\begin{array}{c}\text { Frs } \\
\mathbf{m} / \mathbf{m i n}\end{array}$ & $\begin{array}{c}\mathbf{C E} \\
\mathbf{K g} / \mathbf{C O 2}\end{array}$ & Pre \\
\hline $\mathbf{1}$ & $\mathbf{C E}$ & 2.37 & 97.51 & 3.28 & 148.36 & 0.2249 & 0.85 \\
$\mathbf{2}$ & Pre & 2.08 & 101.64 & 2.87 & 141.72 & 0.4218 & 0.13 \\
$\mathbf{3}$ & $\mathbf{C E} \boldsymbol{\&}$ Pre & 2.16 & 99.52 & 3.09 & 146.61 & 0.2984 & 0.55 \\
\hline
\end{tabular}

Through the comparison of the above hobbing optimization results, the following conclusions can be obtained.

1) When carbon emission $\mathbf{C E}$ is the optimization target, the Frr, Ffr and Frs are higher than those of scheme 2 and 3, while the Rrs is lower.

2) When the comprehensive evaluation of gear precision Pre is taken as the optimization objective, the optimized process parameters are lower than those of scheme 1 and scheme 3 except the Rrs. The feed rate Frr of rough rolling is $87.76 \%$ of scheme 1 , the feed rate Ffr of fine rolling is $87.50 \%$, and the speed Frs of fine rolling is $95.52 \%$.

3) When CE \& Pre was optimized, all the optimized process parameters were between scheme 1 and scheme 2, and Rrs was 1.02 times of scheme 1 , which was $97.91 \%$ of the corresponding parameters in scheme 2; Frr, Ffr and Frs are $91.14 \%, 94.21 \%$ and $98.82 \%$ of scheme 1 , respectively.

4) Among all the process parameters, Frr and Ffr have significant influence on CE, and Frs has the greatest influence on Pre.

\section{CONCLUSION}

NC gear hobbing machine tools face the problems of too conservative processing parameters and low effective utilization rate. As a result, their performance is not fully utilized in the manufacturing process. This paper focuses on the optimization of NC gear hobbing process parameters with carbon emission and precision as the goal, comprehensively analyzes the related influencing factors and designs related experiments using the Box-Behnken Design method. Taking rough rolling feed rate, rough rolling speed, fine rolling feed rate and fine rolling speed as input, carbon emissions and various accuracy indicators as output, a BP neural network prediction model is established, and the model is solved through the PSO algorithm. Finally, the corresponding process parameters under different optimization goals are obtained. This article has a certain enlightenment in the optimization of gear hobbing process parameters and provides a certain idea for the low-carbon optimization of the machining process.

\section{REFERENCES}

[1] Newman S T , Nassehi A, Imani-Asrai R , et al. Energy efficient process planning for $\mathrm{CNC}$ machining[J]. Cirp Journal of Manufacturing Science \& Technology, 2012, 5( 2):127-136. https://doi.org/10.1016/j.cirpj.2012.03.007

[2] Velchev S, Kolev I, Ivanov K, et al. Empirical models for specific energy consumption and optimization of cutting parameters for minimizing energy consumption during turning[J]. Journal of Cleaner Production, 2014, 80(oct.1):139-149.

https://doi.org/10.1016/j.jclepro.2014.05.099

[3] Kant G, Sangwan K S . Prediction and optimization of machining parameters for minimizing power consumption and surface roughness in machining[J]. Journal of Cleaner Production, 2014, 83(nov.15):151-164. https://doi.org/10.1016/j.jclepro.2014.07.073

[4] Campatelli G , Lorenzini L, Scippa A . Optimization of process parameters using a Response Surface Method for minimizing power consumption in the milling of carbon steel[J]. Journal of Cleaner Production, 2014, 66:309-316.

https://doi.org/10.1016/j.jclepro.2013.10.025

[5] Bilga P S , Singh S, Kumar R. Optimization of energy consumption response parameters for turning operation using Taguchi method[J]. Journal of Cleaner Production, 2016:1406-1417.

https://doi.org/10.1016/j.jclepro.2016.07.220

[6] Kumar R , Bilga P S , Singh S . Multi objective optimization using different methods of assigning weights to energy consumption responses, surface roughness and material removal rate during rough turning operation[J]. Journal of Cleaner Production, 2017, 164(oct.15):45-57.

https://doi.org/10.1016/j.jclepro.2017.06.077

[7] Sun, Shouli, Wang, et al. Prediction and optimization of hobbing gear geometric deviations $[\mathrm{J}]$. Mechanism and Machine Theory: Dynamics of Machine Systems Gears and Power Trandmissions Robots and Manipulator Systems Computer-Aided Design Methods, 2018, 120:288-301.

https://doi.org/10.1016/j.mechmachtheory.2017.09.002

[8] Zhang Z, Kusiak A, Zeng Y, et al. Modeling and optimization of a wastewater pumping system with data-mining methods[J]. Applied Energy, 2016, 164(feb.15):303-311. https://doi.org/10.1016/j.apenergy.2015.11.061

[9] Xiao Q, Li C, Tang Y, et al. A knowledge-driven method of adaptively optimizing process parameters for energy efficient turning[J]. Energy, 2018. https://doi.org/10.1016/j.energy.2018.09.191

[10] 钟健, 阎春平, 曹卫东, 等. 基于 BP神经网络和FPA的高速干切滚齿 工艺参数低碳优化决策 $[J]$. 工程设计学报, 2017(4).(in Chinese)

[11] 吕景祥, 唐任仲, 郑军. 数据驱动的车削和钻削加工能耗预测 [J]. 计 算机集成制造系统, 2020(8). (in Chinese)

[12] 李聪波, 崔龙国, 刘飞, 等. 基于广义边界的机械加工系统碳排放量 化方法[J]. 计算机集成制造系统, 2013, 19(9):2229-2236. (in Chinese) 